hep-th/0210196

\title{
Conformal and Superconformal Mechanics Revisited
}

\author{
E. Ivanov, $\mathrm{A}$ S. Krivonos \\ Bogoliubov Laboratory of Theoretical Physics, JINR, \\ 141980 Dubna, Moscow Region, Russia \\ and \\ J. Niederle团 \\ Institute of Physics, Academy of Sciences of the Czech Republic, \\ Prague 8, CZ 182 21, Czech Republic
}

\begin{abstract}
We find, at the Lagrangian off-shell level, the explicit equivalence transformation which relates the conformal mechanics of De Alfaro, Fubini and Furlan to the conformal mechanics describing the radial motion of the charged massive particle in the Bertotti-Robinson $\mathrm{AdS}_{2} \times$ $S^{2}$ background. Thus we demonstrate the classical equivalence of these two systems which are usually regarded as essentially different "old" and "new" conformal mechanics models. We also construct a similar transformation for $N=2, S U(1,1 \mid 1)$ superconformal mechanics in $N=2$ superfield formulation. Performing this transformation in the action of the $N=2$ superconformal mechanics, we find an off-shell superfield action of $N=2$ superextension of Bertotti-Robinson particle. Such an action has not been given before. We show its on-shell equivalence to the $\mathrm{AdS}_{2}$ superparticle action derived from the spontaneous partial breaking of $S U(1,1 \mid 1)$ superconformal symmetry treated as the $N=2 \mathrm{AdS}_{2}$ supersymmetry.
\end{abstract}

*eivanov@thsun1.jinr.ru

${ }^{\dagger}$ krivonos@thsun1.jinr.ru

‡niederle@fzu.cz 


\section{Introduction}

Conformal mechanics [1] and its superconformal extensions [2, 3] were first introduced and studied as the simplest (one-dimensional) models of (super)conformal field theory. Recently, there was a revival of interest in these models in the context of the AdS/CFT correspondence [4, 5, 6] and the physics of black holes. It was argued [7] (see also [8]-[12]) that the so-called "relativistic" generalizations of (super)conformal mechanics are candidate for the conformal field theory dual to $\mathrm{AdS}_{2}$ (super)gravity in the $\mathrm{AdS}_{2} / \mathrm{CFT}_{1}$ version of the above correspondence. The simplest model of that kind is a particle evolving on the $\mathrm{AdS}_{2} \times S^{2}$ background (the Bertotti-Robinson metric [13]) which describes the near-horizon geometry of $d=4$ Reissner-Nordström black hole. The action (or Hamiltonian) of the standard ("nonrelativistic") conformal mechanics is recovered from the worldline action (or Hamiltonian) of this model in the large black-hole mass approximation.

Both the "old" and "new" (super)conformal mechanics models respect the same (super)conformal symmetry, though realized differently in either cases. This suggests that these models can in fact be equivalent to each other. The aim of the present paper is to demonstrate, on the simple examples of one-field conformal mechanics and its $N=2$ superextension, that such an equivalence indeed takes place and is valid off-shell. We explicitly find the equivalence transformation of the time variable and involved fields which maps the "old" conformal mechanics actions on the actions of the $\mathrm{AdS}_{2}$ (super)particle and vice versa. This off-shell equivalence holds at any finite and non-zero value of the $\mathrm{AdS}_{2}$ radius. Crucial for establishing this relation is the treatment of (super)conformal mechanics models as a sort of $d=1$ sigma models on the cosets of $d=1$ conformal group $S L(2, R) \sim S O(1,2)$ and its superextensions [15, 16] (see also [17]). We demonstrate that the "new" (super)conformal models correspond to another choice of these cosets as compared to the "old" ones and show how to construct the relevant worldline actions in the spirit of the recent papers [18, 19]. Comparing the appropriate cosets, we establish the desired equivalence transformation between two classes of (super)conformal mechanics models. In the superconformal case we limit our consideration to the simplest example of $N=2$ superconformal mechanics [2, 3, 16], but our arguments are valid also for higher $N$ cases treated in the nonlinear realizations approach of refs. [15, 16, 17]. For the example considered we find an off-shell $N=2$ superconformal action describing the radial motion of $N=2$ superextension of the Bertotti-Robinson particle. Such an action has not been known before. We show its on-shell equivalence to the $\mathrm{AdS}_{2}$ superparticle action which derives from the spontaneous partial breaking of $S U(1,1 \mid 1)$ superconformal symmetry regarded as the $N=2 \mathrm{AdS}_{2}$ supersymmetry.

\section{Two versions of conformal mechanics}

We start by recalling basics of the bosonic case. The conformal mechanics model of [1] is described by the following worldline action

$$
S=\frac{1}{2} \int d t\left(\mu \dot{x}^{2}-\frac{\gamma}{x^{2}}\right)
$$

\footnotetext{
${ }^{1}$ The issue of equivalence of various conformally invariant $d=1$ models in the Hamiltonian formalism was addressed in [14.
} 
which corresponds to the Hamiltonian

$$
H=\frac{p^{2}}{2 \mu}+\frac{\gamma}{2 x^{2}}
$$

The action (2.1) is invariant under the $d=1$ conformal transformations

$$
\delta t=a+b t+c t^{2} \equiv a(t), \quad \delta x=\frac{1}{2} \dot{a} x=\frac{1}{2}(b+2 c t) x
$$

which form the $S O(1,2)$ group algebra:

$$
i[P, D]=-P, i[K, D]=K, i[P, K]=-2 D .
$$

The generators $P, D$ and $K$ are, respectively, those of translations, dilatations and special conformal transformations and correspond to the parameters $a, b$ and $c$ in (2.3). The Hamiltonian (2.2) is the translation operator in the canonical formalism. It is straightforward to construct the canonical charges corresponding to dilatations and conformal transformations. It was argued in [1] that in the quantum case the true Hamiltonian is a combination of the above $H$ and the conformal generator, such that it generates a compact $S O(2)$ subgroup of $S O(1,2)$.

The "new" conformal mechanics model was proposed in [7] as a model of a charged massive particle moving in the $\mathrm{AdS}_{2} \times S^{2}$ Bertotti-Robinson (BR) background

$$
\begin{aligned}
d s^{2} & =-(2 R / r)^{4} d \tau^{2}+(2 R / r)^{2} d r^{2}+R^{2} d \Omega^{2} \\
A & =(2 R / r)^{2} d \tau
\end{aligned}
$$

Here, the parameter $R$ can be interpreted as the $\mathrm{AdS}_{2}$ radius (equal to that of the sphere $\left.S^{2}\right), d \Omega^{2}=d \theta^{2}+\sin ^{2} \theta d \varphi^{2}$ is the $S O(3)$ invariant metric on $S^{2}$ and $A$ is the expression for the related Maxwell field in the coupled Einstein-Maxwell theory to which the BR background (2.5) provides a solution. The static-gauge action of the particle and the corresponding canonical Hamiltonian are as follows

$$
\begin{aligned}
S & =\int(q A-\tilde{\mu} \sqrt{-G(\tau)} d \tau) \\
& =\int d \tau(2 R / r)^{2}\left[q-\mu \sqrt{1-(2 R / r)^{-2} \dot{r}^{2}-R^{2}(2 R / r)^{-4}\left(\dot{\theta}^{2}+\sin ^{2} \theta \dot{\varphi}^{2}\right)}\right] \\
H & =(2 R / r)^{2}\left[\sqrt{\mu^{2}+\left(r^{2} p_{r}^{2}+4 L^{2}\right) / 4 R^{2}}-q\right] \\
L^{2} & \equiv p_{\theta}^{2}+\sin ^{-2} \theta p_{\varphi}^{2}
\end{aligned}
$$

The action (2.6) is invariant under the field-dependent $d=1$ conformal transformations

$$
\delta \tau=a(\tau)+c\left(1 / 16 R^{2}\right) r^{4}, \quad \delta r=\frac{1}{2} \dot{a} r, \quad \delta \theta=\delta \varphi=0 .
$$

As in the case of "old" conformal mechanics, in this case it is straightforward to find the canonical Noether charges generating the "active" form of the conformal transformations (2.9) for the involved fields [7]. 
In the limit $R \rightarrow \infty, \tilde{\mu}-q \rightarrow 0, R^{2}(q-\tilde{\mu}) \neq \infty$ the action (2.6) and Hamiltonian (2.7) go over into (2.1) and (2.2) with $x \equiv r$ and

$$
\mu=\tilde{\mu}, \quad \gamma=8 R^{2}(\mu-q)+4 L^{2} / \mu .
$$

The "new" conformal mechanics was interpreted in [7]-[11] as a "relativistic" generalization of the "old" one just in this sense (the latter being a limiting case of the former).

The purely $\mathrm{AdS}_{2}$ part of (2.6), (2.7) corresponds to "freezing" the $S^{2}$ angles, $\theta=$ const, $\varphi=$ const, $L^{2}=0$, and so describes the radial motion of the $\operatorname{AdS}_{2} \times S^{2}$ particle. In what follows we shall be interested just in this reduced system which is in itself invariant under the conformal transformations of $\tau$ and $r$ defined in (2.9).

\section{Conformal mechanics models as nonlinear realiza- tions of $S O(1,2)$}

It was shown in [15] that both the action (2.1) and equation of motion of the conformal mechanics of ref. [1] admit a transparent geometric interpretation in terms of left-invariant Cartan 1-forms on the group $S O(1,2)$. Now we are going to show that both the action (2.6) (restricted to the radial motion) and non-standard conformal transformations (2.9) can be also straightforwardly recovered from the same coset space approach.

We start by recalling some basic points of ref. [15.

One proceeds from a nonlinear realization of the $S O(1,2)$ group acting as left shifts on the element

$$
g=e^{i t P} e^{i u(t) D} e^{i \lambda(t) K},
$$

where $u(t)$ and $\lambda(t)$ are the Goldstone fields for the dilatation and special conformal generators. The $S O(1,2)$ left shifts induce for $t, q(t)$ and $\lambda(t)$ the following transformations

$$
\delta t=a+b t+c t^{2}, \quad \delta u=b+2 c t, \delta \lambda=c e^{u} .
$$

Next one defines the Cartan forms

$$
\begin{aligned}
& g^{-1} d g=i \omega_{P} P+i \omega_{D} D+i \omega_{K} K, \\
& \omega_{P}=e^{-u} d t, \quad \omega_{D}=d u-2 e^{-u} \lambda d t, \\
& \omega_{K}=d \lambda+e^{-u} \lambda^{2} d t-\lambda d u,
\end{aligned}
$$

which by construction are invariants of the transformations (3.2). Using this fact, the coset field $\lambda(t)$ can be covariantly eliminated by imposing the inverse Higgs [20] constraint

$$
\omega_{D}=0 \Rightarrow \lambda=\frac{1}{2} e^{u} \dot{u}
$$

The manifestly invariant worldline action

$$
\begin{aligned}
S & =-\frac{1}{2} \int\left(\mu \nu^{2} \omega_{k}+\gamma \nu^{-2} \omega_{P}\right) \\
& =\frac{1}{2} \int d t\left(\frac{1}{4} \mu \nu^{2} e^{u} \dot{u}^{2}-\gamma \nu^{-2} e^{-u}\right)
\end{aligned}
$$


where $\nu([\nu]=-1)$ is a normalization constant, is just the "old" conformal mechanics action (2.1) upon the identification

$$
x(t)=\nu e^{1 / 2 u(t)} .
$$

The transformations (3.2), being rewritten through $x(t)$, coincide with (2.3). Note that one can use in (3.5) the 1 -forms with $u(t)$ and $\lambda(t)$ as independent fields. Then the constraint (3.4) arises as the equation of motion for $\lambda$.

The equation of motion for $x(t)$ or $u(t)$ following from (2.1) or (3.5) was interpreted in [15] as the equation for geodesics on $S O(1,2)$. They can be time-like, space-like or null, depending on the choice of the parameters $\gamma$ and $\mu$.

The basis (2.4) in the algebra $s o(1,2)$ can naturally be called "conformal" as it implies the standard $d=1$ conformal transformations for the time $t$. Now we pass to another basis in the same algebra

$$
\hat{K}=m K-\frac{1}{m} P, \hat{D}=m D,
$$

with $m$ being a parameter of the dimension of mass. This choice will be referred to as the "AdS basis" for a reason soon to be made clear.

The conformal algebra (2.4) in the AdS basis (3.7) reads

$$
i[P, \hat{D}]=-m P, i[\hat{K}, \hat{D}]=2 P+m \hat{K}, i[P, \hat{K}]=-2 \hat{D} .
$$

An element of $S O(1,2)$ in the AdS basis is defined to be

$$
g=e^{i y P} e^{i \phi(y) \hat{D}} e^{i \Omega(y) \hat{K}}
$$

Now we are in a position to explain the motivation for the nomenclature "AdS basis" . With the choice (3.1), the group parameters $t$ and $u(t)$ parameterize the coset of $S O(1,2)$ over conformal generator $K$. So they are $d=1$ analogs of the standard $d=4$ Minkowski space-time 4-coordinate and dilaton which parameterize the coset of the $d=4$ conformal group $S O(2,4)$ over the semi-direct product of Lorentz group $S O(1,3)$ and the commuting abelian subgroup spanned by generators of $d=4$ conformal boosts $K_{m}$. At the same time, the generator $\hat{K}$ (3.7) can be shown to correspond to an $S O(1,1)$ subgroup of $S O(1,2)$. Thus the parameters $y$ and $\phi(y)$ in (3.9) parameterize the coset $S O(1,2) / S O(1,1)$, i.e. $\operatorname{AdS}_{2}$. The parameterization (3.9) of $\mathrm{AdS}_{2}$ is a particular case of the so-called "solvable subgroup parameterization" of the AdS spaces [21. The $d=4$ analog of this parameterization is the parameterization of the $\mathrm{AdS}_{5}$ space in such a way that its coordinates are still parameters associated with the 4-translation and dilatation generators $P_{m}, D$ of $S O(2,4)$, while it is the subgroup $S O(1,4)$ with the algebra $\propto\left\{P_{m}-K_{m}, s o(1,3)\right\}$ which is chosen as the stability subgroup 21].

The difference in the geometric meanings of the coordinate pairs $(t, u(t))$ and $(y, \phi(y))$ is manifested in their different transformation properties under the same $d=1$ conformal transformations. Left shifts of the $S O(1,2)$ group element in the parameterization (3.9) induce the following transformations

$$
\delta y=a(y)+\frac{1}{m^{2}} c e^{2 m \phi}, \quad \delta \phi=\frac{1}{m} \dot{a}(y)=\frac{1}{m}(b+2 c y), \quad \delta \Omega=\frac{1}{m} c e^{m \phi} .
$$

We observe the modification of the special conformal transformation of $y$ by a field-dependent term, just as in eq. (2.9). 
The relevant left-invariant Cartan forms are given by the following expressions

$$
\begin{aligned}
\hat{\omega}_{D} & =\frac{1+\Lambda^{2}}{1-\Lambda^{2}} d \phi-2 \frac{\Lambda}{1-\Lambda^{2}} e^{-m \phi} d y, \\
\hat{\omega}_{P} & =\frac{1+\Lambda^{2}}{1-\Lambda^{2}} e^{-m \phi} d y-2 \frac{\Lambda}{1-\Lambda^{2}} d \phi, \\
\hat{\omega}_{K} & =m \frac{\Lambda}{1-\Lambda^{2}}\left(\Lambda e^{-m \phi} d y-d \phi\right)+\frac{d \Lambda}{1-\Lambda^{2}},
\end{aligned}
$$

where

$$
\Lambda=\tanh \Omega .
$$

As in the previous realization, the field $\Lambda(y)$ can be eliminated by imposing the inverse Higgs constraint

$$
\hat{\omega}_{D}=0 \Rightarrow \partial_{y} \phi=2 e^{-m \phi} \frac{\Lambda}{1+\Lambda^{2}},
$$

whence $\Lambda$ is expressed in terms of $\phi$ :

$$
\Lambda=\partial_{y} \phi e^{m \phi} \frac{1}{1+\sqrt{1-e^{2 m \phi}\left(\partial_{y} \phi\right)^{2}}} .
$$

The $S O(1,2)$ invariant distance on $\mathrm{AdS}_{2}$ can be defined, prior to imposing any constraints, as

$$
d s^{2}=-\hat{\omega}_{P}^{2}+\hat{\omega}_{D}^{2}=-e^{-2 m \phi} d y^{2}+d \phi^{2} .
$$

Making the redefinition

$$
U=e^{-m \phi},
$$

it can be cast into the standard BR form

$$
d s^{2}=-U^{2} d y^{2}+\left(1 / m^{2}\right) U^{-2} d U^{2},
$$

with $1 / m$ as the inverse $\mathrm{AdS}_{2}$ radius,

$$
\frac{1}{m}=R
$$

One more change of variable

$$
U=(2 R / r)^{2}, \quad y \equiv \tau
$$

brings the distance just to the form (2.5) (with the $S^{2}$ part neglected). The modified conformal transformations (3.10) become just (2.9) after these redefinitions.

The action (2.6) can now be easily constructed from the Cartan forms (3.11) which, after substituting the inverse Higgs expression for $\Lambda$, eq. (3.14), read

$$
\begin{aligned}
& \hat{\omega}_{P}=e^{-m \phi} \sqrt{1-e^{2 m \phi}\left(\partial_{y} \phi\right)^{2}} d y, \\
& \hat{\omega}_{K}=-\frac{m}{2} e^{-m \phi}\left(1-\sqrt{1-e^{2 m \phi}\left(\partial_{y} \phi\right)^{2}}\right) d y+\text { Total derivative } \times d y .
\end{aligned}
$$

The invariant action reads

$$
S=-\int\left(\tilde{\mu} \hat{\omega}_{P}-q e^{-m \phi}\right)=-\int d y e^{-m \phi}\left(\tilde{\mu} \sqrt{1-e^{2 m \phi}\left(\partial_{y} \phi\right)^{2}}-q\right) .
$$


After the above field redefinitions it is recognized as the radial-motion part of the "new" conformal mechanics action (2.6). Note that the second term in (3.20) is invariant under (3.10) up to a total derivative in the integrand. The action can be rewritten in a manifestly invariant form (with a tensor Lagrangian) by using the explicit expression for $\hat{\omega}_{K}$ in (3.19)

$$
S=\int\left[(q-\tilde{\mu}) \hat{\omega}_{P}-(2 / m) q \hat{\omega}_{K}\right] .
$$

Note that, like in the previous case, in (3.21) one can use the Cartan forms with $\Lambda(y)$ as an independent field. Then the inverse Higgs expression (3.14) can be reproduced as the equation of motion for $\Lambda(y)$ (with $\tilde{\mu} \neq 0$ ).

Now we are approaching the major point. We see that the "old" and "new" conformal mechanics models are associated with two different nonlinear realizations of the same $d=1$ conformal group $S O(1,2)$ corresponding, respectively, to the two different choices (3.1) and (3.9) of the parameterization of the group element. The invariant actions in both cases can be written as integrals of linear combinations of the left-invariant Cartan forms. But the latter cannot depend on the choice of parameterization. Then the actions (3.5) and (3.21) should in fact coincide with each other up to a redefinition of the free parameters of the actions. Thus two conformal mechanics models are equivalent modulo redefinition of the involved time coordinate and field. This statement should be contrasted with the previous view of the "old" conformal mechanics model as a "non-relativistic" approximation of the "new" one.

To find the relation between actions (3.5) and action (3.21), we first write the relations between Cartan forms in two bases,

$$
\omega_{K}=m \hat{\omega}_{K}, \quad \omega_{P}=\hat{\omega}_{P}-\frac{1}{m} \hat{\omega}_{K},
$$

which follow from the definition (3.7). Then we substitute these relations into (3.5) and compare the resulting action of the "old" conformal mechanics (thus rewritten in the parameterization (3.9) ) with the action (3.21). We find that the two actions coincide with each other, provided that their parameters are related as

$$
\gamma=2 \nu^{2}(\tilde{\mu}-q), \quad \mu=2\left(R^{2} / \nu^{2}\right)(\tilde{\mu}+q) .
$$

Taking into account that (3.5) is invariant under rescalings

$$
\gamma \rightarrow \gamma l, \quad \mu \rightarrow \mu l^{-1}, \quad \nu \rightarrow \nu l^{1 / 2}
$$

one can choose, without loss of generality, the "gauge"

$$
\nu^{2}=4 R^{2},
$$

in which

$$
\gamma=8 R^{2}(\tilde{\mu}-q), \quad \mu=\frac{1}{2}(\tilde{\mu}+q) .
$$

The same result can be obtained by directly performing in (2.1) or (3.5) the change of variables relating the conformal and AdS bases of the $d=1$ conformal group. This transformation can be found by comparing the parameterizations (3.1) and (3.9), with making use of the identity

$$
e^{i t \hat{K}}=e^{-\frac{i}{m}(\tanh t) P} e^{-2 i(\ln \cosh t) D} e^{i m(\tanh t) K},
$$


which can be checked using (3.7), (3.8). The sought change of coordinates is as follows

$$
t=y-\frac{1}{m} e^{m \phi} \Lambda, u=m \phi+\ln \left(1-\Lambda^{2}\right), \lambda=m \Lambda .
$$

These relations, together with (3.25) and the inverse Higgs expressions (3.4), (3.14) substituted for $\lambda, \Lambda$, fully fix the equivalence map between the "old" and "new" conformal mechanics.

Finally, two remarks are in order.

First, for deriving the map (3.27) it was essential that in our way of constructing the $\mathrm{AdS}_{2}$ particle action in terms of Cartan one-forms we started not just from the coset $S O(1,2) / S O(1,1) \sim \mathrm{AdS}_{2}$, but from the whole $S O(1,2)$ group space, including the parameter $\Lambda(y)$ associated with the $S O(1,1)$ generator $\hat{K}$. This parameter has been eventually covariantly traded for $\phi, \partial_{y} \phi$ according to (3.14), but just its presence allowed us to equate the elements of $S O(1,2)$ in the parameterizations (3.1), (3.9) and to derive the relations (3.27). It would be difficult to guess these relations, while constructing the $\mathrm{AdS}_{2}$ particle action in the conventional way, just proceeding from a $d=1$ pullback of the $\mathrm{AdS}_{2}$ metric (3.15).

Secondly, the above map is well-defined at $R \neq \infty, R \neq 0$, i.e. it is one-to-one only for non-zero and finite value of the $\mathrm{AdS}_{2}$ radius. The action (2.6) is still well defined at $m=0(R=\infty)$, in this limit it becomes the static-gauge action of the massive particle in $d=2$ Minkowsky space. However, such an action does not respect $d=1$ conformal symmetry, its only symmetries are the $d=2$ translation and $S O(1,1)$ rotation ones. Thus any link with the conformal mechanics turns out to be lost in this limit.

\section{$4 \mathrm{~N}=2$ superconformal mechanics}

The previous consideration may be generalized to the supersymmetry case. As the simplest example we consider in this Section $N=2$ superconformal mechanics [2, 13, 16]. Like its bosonic prototype, $N=2$ superconformal mechanics has a natural description within the coset approach [16.

The starting point is the $s u(1,1 \mid 1)$ superalgebra which includes, apart from the $s o(1,2)$ generators (2.4), those of Poincarè $\{Q, \bar{Q}\}$ and conformal $\{S, \bar{S}\}$ supersymmetries and the $U(1)$ generator $U$. In the conformal basis the non-vanishing (anti)commutators read:

$$
\begin{aligned}
& \{Q, \bar{Q}\}=-2 P,\{Q, \bar{S}\}=-2 D+2 U,\{S, \bar{S}\}=-2 K,\{S, \bar{Q}\}=-2 D-2 U, \\
& i\left[P,\left(\frac{S}{S}\right)\right]=-\left(\frac{Q}{Q}\right), i\left[K,\left(\frac{Q}{Q}\right)\right]=\left(\frac{S}{\bar{S}}\right), \\
& i\left[D,\left(\frac{Q}{Q}\right)\right]=\frac{1}{2}\left(\frac{Q}{Q}\right), i\left[D,\left(\frac{S}{S}\right)\right]=-\frac{1}{2}\left(\frac{S}{S}\right), \\
& i\left[U,\left(\frac{Q}{Q}\right)\right]=\frac{1}{2}\left(-\frac{Q}{Q}\right), i\left[U,\left(\frac{S}{S}\right)\right]=\frac{1}{2}\left(\begin{array}{r}
S \\
-S
\end{array}\right) .
\end{aligned}
$$

The standard realization of $S U(1,1 \mid 1)$ as the spontaneously broken $d=1, N=2$ superconformal group is set up by left multiplications of the coset

$$
g=e^{i t P} e^{\theta Q+\overline{\theta Q}} e^{i q D} e^{i \lambda K} e^{\psi S+\bar{\psi} \bar{S}},
$$


where $(t, \theta, \bar{\theta})$ are coordinates of $d=1, N=2$ superspace and the remaining coset parameters are superfields given on this superspace. The Poincarè supersymmetry transformations have the form

$$
\delta t=-i(\varepsilon \bar{\theta}+\bar{\varepsilon} \theta), \quad \delta \theta=\varepsilon, \quad \delta \bar{\theta}=\bar{\varepsilon},
$$

while the superconformal ones are given by

$$
\delta t=-i t(\epsilon \bar{\theta}+\bar{\epsilon} \theta), \delta \theta=\epsilon(t+i \theta \bar{\theta}), \delta \bar{\theta}=\bar{\epsilon}(t-i \theta \bar{\theta}), \delta q=-2 i(\epsilon \bar{\theta}+\bar{\epsilon} \theta) .
$$

To single out the minimal set of coordinates and Goldstone superfields, as well as to construct manifestly covariant dynamical equations of motion for the latter, we first define the Cartan 1-forms

$$
\begin{aligned}
& \omega_{P}=e^{-q} d \tilde{t}, \omega_{D}=d q-2 e^{-q} \lambda d \tilde{t}+2 i e^{-\frac{1}{2} q}(\psi d \bar{\theta}+\bar{\psi} d \theta) \\
& \omega_{K}=d \lambda+\lambda^{2} e^{-q} d \tilde{t}-\lambda d q-2 i e^{-\frac{1}{2} q} \lambda(\psi d \bar{\theta}+\bar{\psi} d \theta)+i(\psi d \bar{\psi}+\psi d \bar{\psi}), \\
& \omega_{Q}=e^{-\frac{1}{2} q} d \theta-e^{-q} d \tilde{t} \psi, \omega_{S}=d \psi-\frac{1}{2} \psi\left(d q-2 e^{-q} \lambda d \tilde{t}\right)-e^{-\frac{1}{2} q}(\lambda+i \psi \bar{\psi}) d \theta, \\
& \omega_{\bar{Q}}=e^{-\frac{1}{2} q} d \bar{\theta}-e^{-q} d \tilde{t} \bar{\psi}, \omega_{\bar{S}}=d \bar{\psi}-\frac{1}{2} \bar{\psi}\left(d q-2 e^{-q} \lambda d \tilde{t}\right)-e^{-\frac{1}{2} q}(\lambda-i \psi \bar{\psi}) d \bar{\theta}
\end{aligned}
$$

where

$$
d \tilde{t}=d t+i(\theta d \bar{\theta}+\bar{\theta} d \theta)
$$

Then, following ref. [16], we subject them to the appropriate covariant constraints.

The first kind of constraints is the inverse Higgs ones [20]:

$$
\omega_{D}=0 \Rightarrow\left\{\begin{array}{l}
\dot{q}=2 e^{-q} \lambda \\
D q=2 i e^{-\frac{1}{2} q} \bar{\psi} \\
\bar{D} q=2 i e^{-\frac{1}{2} q} \psi
\end{array}\right.
$$

where

$$
D=\frac{\partial}{\partial \theta}+i \bar{\theta} \partial_{t}, \bar{D}=\frac{\partial}{\partial \bar{\theta}}+i \theta \partial_{t},\{D, \bar{D}\}=2 i \partial_{t}
$$

As in the bosonic case, they are purely kinematical and serve to covariantly express the redundant Goldstone superfields $\lambda, \psi, \bar{\psi}$ as derivatives of the only essential Goldstone superfield, the dilaton $q(t, \theta, \bar{\theta})$.

The equations of motion are produced by the extra covariant condition [16]

$$
\sqrt{\mu} \nu^{2} \omega_{S}=-i \sqrt{\gamma} \omega_{Q}
$$

and they read

$$
[D, \bar{D}] Y=\frac{2}{\nu^{2}} \sqrt{\frac{\gamma}{\mu}} \frac{1}{Y}, \quad Y=e^{\frac{1}{2} q} .
$$

In contrast to the bosonic case, the lagrangian density of the action of $N=2$ superconformal mechanics cannot be directly constructed out of the Cartan forms: it is not strictly invariant under superconformal transformations and in this respect resembles the Wess-Zumino-Witten or Chern-Simons type Lagrangians. It has the following form

$$
S_{\text {conf }}=\int d t d^{2} \theta\left(\frac{\mu \nu^{2}}{2} D Y \bar{D} Y+\sqrt{\mu \gamma} \ln Y\right)
$$


and one can check that the Lagrangian in it is shifted by a total derivative under (4.4). Such behavior of the Lagrangian is a key feature of most supersymmetric systems with partial breaking of global supersymmetries (see e.g. [22]). This property makes the construction of the invariant minimal superfield actions in superconformal mechanics a rather complicated problem as compared to the pure bosonic case.

The first component of superfield $q$ is just $\left.u(t) \equiv q(t, \theta)\right|_{\theta=0}$ and the bosonic part of the action (4.10) coincides with the bosonic action (3.5) (after eliminating the auxiliary field $\left.[D, \bar{D}] q\right|_{\theta=0}$ by its equation of motion).

Now we shall consider a supersymmetric extension of the AdS basis (3.7). The only new thing we have to do for this is to make a rescaling of the superconformal generators as $\hat{S}=m S, \hat{S}=m \bar{S}$. The superconformal algebra $s u(1,1 \mid 1)$ in the AdS basis then reads:

$$
\begin{aligned}
& \{Q, \hat{\bar{S}}\}=-2 \hat{D}+2 m U,\{\hat{S}, \hat{\bar{S}}\}=-2 m \hat{K}-2 P,\{\hat{S}, \bar{Q}\}=-2 \hat{D}-2 m U, \\
& i\left[P,\left(\begin{array}{c}
\hat{S} \\
\hat{\bar{S}}
\end{array}\right)\right]=-m\left(\frac{Q}{Q}\right), i\left[\hat{K},\left(\frac{Q}{Q}\right)\right]=\left(\begin{array}{c}
\hat{S} \\
\hat{\bar{S}}
\end{array}\right), i\left[\hat{K},\left(\begin{array}{c}
\hat{S} \\
\hat{\bar{S}}
\end{array}\right)\right]=\left(\frac{Q}{Q}\right), \\
& i\left[\hat{D},\left(\begin{array}{c}
Q \\
\bar{Q}
\end{array}\right)\right]=\frac{m}{2}\left(\frac{Q}{Q}\right), i\left[\hat{D},\left(\begin{array}{c}
\hat{S} \\
\hat{\bar{S}}
\end{array}\right)\right]=-\frac{m}{2}\left(\begin{array}{c}
\hat{S} \\
\hat{\bar{S}}
\end{array}\right) \\
& i\left[U,\left(\begin{array}{c}
\hat{S} \\
\hat{\bar{S}}
\end{array}\right)\right]=\frac{1}{2}\left(\begin{array}{c}
\hat{S} \\
-\hat{\bar{S}}
\end{array}\right), i\left[U,\left(\frac{Q}{Q}\right)\right]=\frac{1}{2}\left(\begin{array}{c}
Q \\
-\bar{Q}
\end{array}\right) .
\end{aligned}
$$

We define the realization of $S U(1,1 \mid 1)$ in the AdS basis by its left action on the coset $S U(1,1 \mid 1) / U(1)$ in the following parameterization:

$$
g=e^{i y P} e^{\theta Q+\bar{\theta} \bar{Q}} e^{i \Phi \hat{D}} e^{i \Omega \hat{K}} e^{\xi \hat{S}+\overline{\bar{\xi}}} .
$$

Left $S U(1,1 \mid 1)$ shift of the coset (4.12) induce the following superconformal transformations for the coset parameters

$$
\begin{aligned}
& \delta y=-i(\epsilon \bar{\theta}+\bar{\epsilon} \theta)\left[m y+e^{m \Phi} \Lambda\right]-i(\epsilon \bar{\xi}+\bar{\epsilon} \xi) e^{\frac{3}{2} m \Phi} \frac{1+\Lambda^{2}}{\sqrt{1-\Lambda^{2}}}, \\
& \delta \theta=m \epsilon\left(y+i \theta \bar{\theta}-\frac{1}{m} e^{m \Phi} \Lambda\right), \delta \bar{\theta}=m \bar{\epsilon}\left(y-i \theta \bar{\theta}-\frac{1}{m} e^{m \Phi} \Lambda\right) \\
& \delta \Phi=-2 i(\epsilon \bar{\theta}+\bar{\epsilon} \theta)-2 i(\epsilon \bar{\xi}+\bar{\epsilon} \xi) e^{\frac{1}{2} m \Phi} \frac{\Lambda}{\sqrt{1-\Lambda^{2}}}, \\
& \delta \xi=\epsilon e^{\frac{1}{2} m \Phi} \sqrt{1-\Lambda^{2}}+i m(\bar{\epsilon} \theta-\epsilon \bar{\theta}) \xi, \delta \bar{\xi}=\bar{\epsilon} e^{\frac{1}{2} m \Phi} \sqrt{1-\Lambda^{2}}-i m(\bar{\epsilon} \theta-\epsilon \bar{\theta}) \bar{\xi} \\
& \delta \Lambda=-i m(\bar{\epsilon} \theta+\epsilon \bar{\theta}) e^{\frac{1}{2} m \Phi} \sqrt{1-\Lambda^{2}}
\end{aligned}
$$

where $\Lambda$ is still defined according to 3.12 as $\Lambda=\tanh \Omega$.

The relevant Cartan form needed to covariantly trade the superfields $\Omega, \xi, \bar{\xi}$ for derivatives of $\Phi$ reads:

$$
\omega_{\hat{D}}=\cosh (2 \Omega) d \Phi-\sinh (2 \Omega) e^{-m \Phi} d \tilde{y}+2 i \cosh (\Omega) e^{-\frac{m}{2} \Phi}(\xi d \bar{\theta}+\bar{\xi} d \theta),
$$

where

$$
d \tilde{y}=d y+i(\theta d \bar{\theta}+\bar{\theta} d \theta)
$$


Putting this form equal to zero, we obtain

$$
\partial_{y} \Phi=2 e^{-m \Phi} \frac{\Lambda}{1+\Lambda^{2}}, D_{y} \Phi=2 i e^{-\frac{m}{2} \Phi} \frac{\sqrt{1-\Lambda^{2}}}{1+\Lambda^{2}} \bar{\xi}, \bar{D}_{y} \Phi=2 i e^{-\frac{m}{2} \Phi} \frac{\sqrt{1-\Lambda^{2}}}{1+\Lambda^{2}} \xi,
$$

where

$$
D_{y}=\frac{\partial}{\partial \theta}+i \bar{\theta} \partial_{y}, \bar{D}_{y}=\frac{\partial}{\partial \bar{\theta}}+i \theta \partial_{y},\left\{D_{y}, \bar{D}_{y}\right\}=2 i \partial_{y}
$$

Now we are able to establish a link between the above two nonlinear realizations. This equivalence transformation is a supersymmetric extension of (3.27). The relations between the coset coordinates can be found by rearranging the exponents from the parameterization (4.2) to (4.12). The explicit form of these relations is as follows

$$
t=y-\frac{1}{m} e^{m \Phi} \Lambda, q=m \Phi+\ln \left(1-\Lambda^{2}\right), \lambda=m \Lambda, \psi=m \xi, \bar{\psi}=m \bar{\xi} .
$$

Now we have all the necessary ingredients for passing from one basis another in the $N=2$ superconformally invariant actions. As the suggestive example, let us rewrite the standard $N=2$ superconformal mechanics action (4.10) in the AdS basis

$$
\begin{aligned}
& S=\frac{1}{2} \int d t d^{2} \theta\left(-\mu \nu^{2} \bar{\psi} \psi+\sqrt{\mu \gamma} q\right)=\frac{1}{2} \int d y d^{2} \theta\left[\frac{1-\Lambda^{2}}{1+\Lambda^{2}}-\frac{1}{m} e^{m \Phi} \partial_{y} \Lambda\right] \\
& \times\left[\frac{1}{4} \mu \nu^{2} m^{2} e^{m \Phi} \frac{\left(1+\Lambda^{2}\right)^{2}}{1-\Lambda^{2}} D_{y} \Phi \bar{D}_{y} \Phi+\sqrt{\mu \gamma}\left(m \Phi+\ln \left(1-\Lambda^{2}\right)\right)\right] .
\end{aligned}
$$

Here

$$
\Lambda=e^{m \Phi} \partial_{y} \Phi \frac{1}{1+\sqrt{1-e^{2 m \Phi}\left(\partial_{y} \Phi\right)^{2}}}, \quad \Lambda^{2}=\frac{1-\sqrt{1-e^{2 m \Phi}\left(\partial_{y} \Phi\right)^{2}}}{1+\sqrt{1-e^{2 m \Phi}\left(\partial_{y} \Phi\right)^{2}}}
$$

as follows from (4.15). From now on we shall use the "gauge" (3.24):

$$
\nu^{2} m^{2}=4 R^{2} m^{2}=4 .
$$

While neglecting the superpotential term (i.e. choosing $\gamma=0$ ) and terms with higher derivatives (those with $\partial_{y} \Lambda$ ), the action is simplified to the form

$$
S=\mu \int d y d^{2} \theta\left[\frac{e^{m \Phi} D_{y} \Phi \bar{D}_{y} \Phi}{1+\sqrt{1-e^{2 m \Phi}\left(\partial_{y} \Phi\right)^{2}}}\right]
$$

which is the product of the first terms in both expressions inside the square brackets in (4.18). One can directly check that the bosonic part of (4.21) just coincides with (3.21) (for $\tilde{\mu}=q$ ) after elimination of the auxiliary field. Using the definition $d^{2} \theta=\bar{D} D$, and suppressing the fermionic fields, one finds

$$
\begin{aligned}
S_{b o s}^{0} & \equiv \int d y L_{0}=\frac{\mu}{2} \int d y\left[\left(\partial_{y} \phi\right)^{2}+F^{2}\right] e^{m \phi}\left(1+\Lambda^{2}\right) \\
& =\mu \int d y\left[\left(\partial_{y} \phi\right)^{2}+F^{2}\right] e^{m \phi} \frac{1}{1+\sqrt{1-e^{2 m \phi}\left(\partial_{y} \phi\right)^{2}}}
\end{aligned}
$$


On shell, i.e. with $F=0$, we obtain

$$
S_{\text {bos }}^{0}=\mu \int d y e^{-m \phi}\left[1-\sqrt{1-e^{2 m \phi}\left(\partial_{y} \phi\right)^{2}}\right] .
$$

The full action (4.18) looks rather complicated and includes terms which could seemingly give rise to higher derivatives in $y$ in the component Lagrangian. Nevertheless, this does not happen. By direct calculation, the remaining contributions to the bosonic part of the off-shell component action are given by

$$
S_{b o s}^{1}=\int d y\left(L_{1}+L_{2}\right)
$$

where

$$
\begin{aligned}
& L_{1}=-\frac{\mu}{4 m}\left[\left(\partial_{y} \phi\right)^{2}+F^{2}\right] e^{3 m \phi} \frac{\left(1+\Lambda^{2}\right)^{4}}{\left(1-\Lambda^{2}\right)^{2}}\left[\partial_{y}^{2} \phi+m\left(\partial_{y} \phi\right)^{2}\right], \\
& L_{2}=-\frac{1}{2} \sqrt{\mu \gamma} F\left\{m-e^{2 m \phi} \frac{\left(1+\Lambda^{2}\right)^{3}}{2\left(1-\Lambda^{2}\right)^{2}}\left[\partial_{y}^{2} \phi+m\left(\partial_{y} \phi\right)^{2}\right]\right\} .
\end{aligned}
$$

After the change of variable

$$
X=\frac{1}{m} e^{m \phi} \Rightarrow \Lambda \equiv \Lambda\left(\partial_{y} X\right)=\partial_{y} X \frac{1}{1+\sqrt{1-\left(\partial_{y} X\right)^{2}}}
$$

the $F$-independent term in (4.25) can be shown to be a full derivative

$$
\sim \partial_{y}^{2} X f\left(\partial_{y} X\right)
$$

Thus (4.24) contributes only to the potential of the auxiliary field $F(y)$. The algebraic equation for the latter which follows from $S_{b o s}=S_{b o s}^{0}+S_{b o s}^{1}$ is easily found to be

$$
F=\frac{m}{4} \sqrt{\frac{\gamma}{\mu}} e^{-m \phi}\left[1+\sqrt{1-e^{2 m \phi}\left(\partial_{y} \phi\right)^{2}}\right] .
$$

After substituting this back into $S_{b o s}$, the latter, modulo a full derivative in the Lagrangian, reads

$$
S_{b o s}=\int d y e^{-m \phi}\left\{\mu\left[1-\sqrt{1-e^{2 m \phi}\left(\partial_{y} \phi\right)^{2}}\right]-\frac{\gamma}{16} m^{2}\left[1+\sqrt{1-e^{2 m \phi}\left(\partial_{y} \phi\right)^{2}}\right]\right\}
$$

which coincides with (3.20) under the identification (3.25).

Thus (4.18) provides a manifestly $N=2$ supersymmetric off-shell form of $N=2$ superconformal extension of the "new" conformal mechanics action (3.20) which describes the radial $\left(\mathrm{AdS}_{2}\right)$ motion of the charged particle in the $\mathrm{BR} \mathrm{AdS}_{2} \times S^{2}$ background. Such a superfield action was not known before. By construction, it is related by the equivalence transformation (4.17) to the "old" $N=2$ superconformal mechanics action (4.10).

As the last topic, we would like to show that the action (4.18) at $\gamma=0$ is equivalent, at least on-shell, to the "standard" action for the superparticle in $\mathrm{AdS}_{2}$ background. The 
latter action can be obtained by dimensional reduction from that of $N=1$ supermembrane in $\mathrm{AdS}_{4}$ background [18, 23] and has the following form:

$$
\tilde{S}=\mu \int d y d^{2} \theta \mathcal{L} \equiv \mu \int d y d^{2} \theta\left[\frac{e^{m \tilde{\Phi}} D_{y} \tilde{\Phi} \bar{D}_{y} \tilde{\Phi}}{1+\sqrt{1+e^{2 m \tilde{\Phi}} D_{y} \bar{D}_{y} \tilde{\Phi} \bar{D}_{y} D_{y} \tilde{\Phi}}}\right],\left.\quad \tilde{\Phi}\right|_{\theta=0}=\left.\Phi\right|_{\theta=0}
$$

This action can be shown to be invariant (up to a shift of the Lagrangian by a full derivative) under the following transformations

$$
\delta \tilde{\Phi}=L \tilde{\Phi}-2 i(\epsilon \bar{\theta}+\bar{\epsilon} \theta)+i e^{m \tilde{\Phi}}\left(\epsilon D_{y} \mathcal{L}+\bar{\epsilon} \bar{D}_{y} \mathcal{L}\right)
$$

where

$$
L=i m \epsilon(\theta \bar{\theta}+2 i y) \frac{\partial}{\partial \theta}-i m \bar{\epsilon}(\theta \bar{\theta}-2 i y) \frac{\partial}{\partial \bar{\theta}}+2 i m(\bar{\epsilon} \theta+\epsilon \bar{\theta}) y \partial_{y} .
$$

These transformations, although being different from (4.13), still constitute a nonlinear realization of the same $N=2$ superconformal group $S U(1,1 \mid 1)$, with $\tilde{\Phi}$ being a Goldstone dilaton superfield.

The purely bosonic part of (4.29) reads

$$
\tilde{S}_{b o s}=\mu \int d y\left[\left(\partial_{y} \phi\right)^{2}+\tilde{F}^{2}\right] e^{m \phi} \frac{1}{1+\sqrt{1-e^{2 m \phi}\left[\left(\partial_{y} \phi\right)^{2}+\tilde{F}^{2}\right]}}
$$

and it obviously coincides on shell, when $F=\tilde{F}=0$, with the bosonic part of (4.18) at $\gamma=0$, viz. $S_{\text {bos }}(\gamma=0)=\int d t\left(L_{0}+L_{1}\right) 2$ Thus it remains to demonstrate that in both actions, 4.18) (at $\gamma=0$ ) and (4.29), the fermionic terms on shell also coincide with each other modulo an equivalence redefinition of the fermionic fields. The proof is based on the following common feature of both actions: after elimination of the auxiliary fields $F$ and $\tilde{F}$ by their equations of motion, the fermionic terms in (4.18) and (4.29) take the following generic form

$$
a_{1}\left(\partial_{y} \psi \bar{\psi}-\psi \partial_{y} \bar{\psi}\right)+a_{2} \psi \partial_{y} \psi \bar{\psi} \partial_{y} \bar{\psi}
$$

Here, $a_{1}$ and $a_{2}$ are some functions of $\phi$ and $\partial_{y} \phi$ which are specific for either actions. The crucial observation is that the invertible redefinition of the fermionic variables

$$
\psi \rightarrow \tilde{\psi}=\sqrt{a_{1}} \psi-\frac{a_{2}}{2 \sqrt{a_{1}}} \psi \partial_{y} \psi \bar{\psi}, \quad \bar{\psi} \rightarrow \widetilde{\bar{\psi}}=\sqrt{a_{1}} \bar{\psi}-\frac{a_{2}}{2 \sqrt{a_{1}}} \psi \partial_{y} \bar{\psi} \bar{\psi}
$$

reduces fermionic terms in both actions to the pure kinetic term

$$
\partial_{y} \tilde{\psi} \widetilde{\bar{\psi}}-\tilde{\psi} \partial_{y} \widetilde{\bar{\psi}}
$$

for arbitrary functions $a_{1}$ and $a_{2}$ (the function $a_{1}$ is assumed to start with a constant, so it is legitimate to divide by it in (4.34) $)$. Keeping in mind that the bosonic parts of the onshell actions are the same, we conclude that on-shell the actions (4.29) and (4.18) coincide with each other, modulo an equivalence redefinition of the fermionic components. It would be instructive to find a superfield redefinition relating $\Phi$ and $\tilde{\Phi}$ and to prove the off-shell equivalence of (4.29) and (4.18).

\footnotetext{
${ }^{2}$ Actually, it is easy to find an invertible relation between the auxiliary fields $F$ and $\tilde{F}$ to show that $\tilde{S}_{b o s}$ and $S_{b o s}(\gamma=0)$ are off-shell equivalent.
} 


\section{Conclusions}

In this paper we have found an equivalence transformation between two nonlinear realizations of the group $S O(1,2)$ : its realization as the spontaneously broken $d=1$ conformal symmetry and the realization as the $\mathrm{AdS}_{2}$ isometry group. This transformation takes the action of the "old" conformal mechanics into the action of the "new" one, viz. the worldline action describing the radial motion of a charged BR particle. Thus these systems, usually treated as two essentially different models of conformal mechanics, prove in fact to be classically equivalent. We extended this equivalence transformation to the case of $N=2$ superconformal mechanics. Being applied to the standard $N=2$ superfield action of the latter, this transformation produces a novel superfield action which provides an $N=2$ superextension of the "new" conformal mechanics action and so describes a radial motion of $N=2$ BR superparticle in a static gauge. Similar equivalence transformations can hopefully be established for higher $N$ superconformal mechanics models in the nonlinear realization approach of refs. 115, 16, 17].

It is very interesting to elaborate on the quantum implications of the equivalence transformation found. We expect that it will allow one to explicitly solve the quantum mechanics of the BR particle and its superextensions in terms of (super)conformal quantum mechanics. Also, it would be worth to extend our consideration to the full BR $\operatorname{AdS}_{2} \times S^{2}$ background and its superextensions, thus taking into account the angular degrees of freedom of the BR (super)particle. The corresponding "old" conformal mechanics action should include a conformally invariant $d=1 S^{2}$ sigma model part. It could naturally appear as a bosonic core of the action of one of possible versions of $N=4$ superconformal mechanics, e.g. associated with the superconformal group $S U(1,1 \mid 2)$.

\section{Acknowledgements}

This work was partially fulfilled during a few collaborative visits of E.A. and S.K. to Institute of Physics in Prague in the framework of the Votruba-Blokhintsev Program. They thank the Directorate of the Institute for hospitality extended to them and the Votruba-Blokhintsev Program for financial support. Their work was also partially supported by an INTAS grant, project No. 00-00254, DFG grant, project No. 436 RUS 113/669, RFBR-DFG grant, project No. 02-02-04002 and RFBR-CNRS grant, project No. 01-02-22005.

\section{References}

[1] V. De Alfaro, S. Fubini, G. Furlan, Nuovo Cim. A 34 (1974) 569.

[2] V. Akulov, A. Pashnev, Teor. Mat. Fiz. 56 (1983) 344.

[3] S. Fubini, E. Rabinovici, Nucl. Phys. B 245 (1984) 17.

[4] J. Maldacena, Adv. Theor. Math. Phys. 2 (1998) 231; hep-th/9711200.

[5] S.S. Gubser, I.R. Klebanov, A.M. Polyakov, Phys. Lett. B 428 (1998) 105; hep-th/9802109. 
[6] E. Witten, Adv. Theor. Math. Phys. 2 (1998) 253; hep-th/9802150.

[7] P. Claus, M. Derix, R. Kallosh, J. Kumar, P.K. Townsend, A. Van Proeyen, Phys. Rev. Lett. 81 (1998) 4553; hep-th/9804177.

[8] R. Kallosh, "Black holes, branes and superconformal symmetry", Proceedings of 2nd Conference on Quantum Aspects of Gauge Theories, Supersymmetry and Unification, Corfu, Greece, 21-26 Sep 1998, pp. 138-168; hep-th/9901095.

[9] R. Kallosh, "Black holes and quantum mechanics", Proceedings of 22nd Johns Hopkins Workshop on Novelties of String Theory, Goteborg, Sweden, 20-22 Aug 1998, pp. 207213 ; hep-th/9902007.

[10] R. Kallosh, J. Kumar, A. Rajaraman, Phys. Rev. D 57 (1998) 6452; hep-th/9712073.

[11] P. Claus, R. Kallosh, J. Kumar, P.K. Townsend, A. Van Proeyen, JHEP 9806 (1998) 004; hep-th/9801206.

[12] G.W. Gibbons, P.K. Townsend, Phys. Lett. B 454 (1999) 187; hep-th/9812034.

[13] B. Bertotti, Phys. Rev. 116 (1959) 1331; I. Robinson, Bull. Acad. Pol. 7 (1959) 351.

[14] S. Cacciatori, D. Klemm, D. Zanon, Class. Quant. Grav. 17 (2000) 1731; hep-th/9910065.

[15] E. Ivanov, S. Krivonos, V. Leviant, J. Phys. A: Math. Gen. 22 (1989) 345.

[16] E. Ivanov, S. Krivonos, V. Leviant, J. Phys. A: Math. Gen. 22 (1989) 4201.

[17] J.A. de Azcarraga, J.M. Izquierdo, J.C. Perez Bueno, P.K. Townsend, Phys. Rev. D 59 (1999) 084015; hep-th/9810230.

[18] F. Delduc, E. Ivanov, S. Krivonos, Phys. Lett. B 529 (2002) 233; hep-th/0111106.

[19] S. Bellucci, E. Ivanov, S. Krivonos, "AdS/CFT Equivalence Transformation", hep-th/0206126.

[20] E. Ivanov, V. Ogievetsky, Teor. Mat. Fiz. 25 (1975) 164.

[21] L. Castellani, A. Ceresole, R. D’Auria, S. Ferrara, P. Fre, M. Trigiante, Nucl. Phys. B 527 (1998) 142; hep-th/9803039.

[22] S. Bellucci, E. Ivanov, S. Krivonos, Nucl. Phys. Proc. Suppl. 102 (2001) 26; hep-th/0103136.

[23] E. Ivanov, S. Krivonos, Phys. Part. Nucl. 32S1 (2001) 35. 